\title{
Les rivières de l'ouest de la France sont-elles seulement des infrastructures naturelles ? Les modèles de gestion à l'épreuve de la directive-cadre sur l'eau
}

\author{
Marie-Anne Germaine ${ }^{1}$, Régis Barraud ${ }^{2}$ \\ 1 Géographe, CNRS, UMR LAVUE 7218 Laboratoire MOSAÏQUES, Université Paris Ouest Nanterre La Défense, 92001 Nanterre, \\ France \\ 2 Géographe, Université de Poitiers, Laboratoire RURALITÉS EA 2252, MSH de Poitiers, 86022 Poitiers, France
}

La longue histoire des hommes et des rivières est marquée par une succession d'aménagements, de démantèlements, d'essais techniques divers... Ces ajustements techniques s'inscrivent dans des visions des cours d'eau qui évoluent selon les époques. Or, nous serions aujourd'hui à une étape charnière. En prenant l'exemple des petites rivières de l'ouest de la France, M.-A. Germaine et R. Barraud examinent les modèles alternatifs à celui de la "rivière aménagée »; ils nous montrent que le «non aménagement » peut être conçu comme un modèle d'aménagement, qui répond à des objectifs dont la négociation sociale est parfois localement conflictuelle.

La Rédaction

\section{Mots-clés :}

environnement ; gouvernance ; cours d'eau ; restauration écologique ; ouest de la France

\begin{abstract}
Résumé - Les nouvelles lois sur l'eau entraînent une multiplication des opérations de restauration écologique des cours d'eau. Qu'ils se traduisent par le désaménagement d'ouvrages ou par la volonté de maintenir des paysages «sauvages » dans les fonds de vallées, ces projets suscitent localement des oppositions et conflits qui témoignent de la diversité des usages et représentations de ces espaces. L'analyse des alternatives proposées au modèle de la rivière aménagée conduit à s'interroger sur les décalages entre les préconisations des services de l'État et les attentes exprimées par les populations locales. À partir de l'étude d'opérations achevées ou en cours dans les petites vallées de l'ouest de la France, des pistes de réflexion pour une meilleure prise en compte des enjeux sociaux et économiques sont proposées pour la mise en œuvre de projets de « rivières vivantes ».
\end{abstract}

\section{Keywords:}

environment; governance; river; ecological restoration; northwestern France

\begin{abstract}
Are the small valleys of Western France natural infrastructures only? The management models under the test of WFD. Since the adoption of the EU Water Framework Directive in 2000, all European waters are to achieve good ecological status by 2015 . The strengthening of water policies involves an increased number of river basin management plans and even of river restoration projects at the local scale. In France the Environment Ministry is promoting a dam removal policy aimed at restoring the ecological continuity of rivers. This policy concerns medium dams and small weirs which were associated with mills. A part of these, however, continue to be used to produce electricity. Simultaneously, river and valley landscapes are drawing new attention involving multiple expectations linked to the valorization of river amenities, which can be at variance with the environmental issue. At local scale, observed conflicts illustrate local water governance difficulties. From the example of river restoration projects which we studied in northwestern France this paper attempts to throw light on different ways and goals of water management. If the regulated river is obsolete, how are rivers to evolve between ecological issues - "wild rivers" - and social objectives - "living rivers" -?
\end{abstract}

Auteur correspondant : M.-A. Germaine ${ }^{1}$, marie-anne.germaine@u-paris10.fr

1 Chercheur associé au GÉOPHEN (UMR LETG 6554 CNRS), Université Caen Basse-Normandie. 
Le démantèlement de seuils et de barrages est devenu en quelques années une mesure phare des programmes de restauration écologique des cours d'eau. Depuis les premières réalisations emblématiques de Kernansquillec et Maisons-Rouges au milieu des années 1990, la gestion des ouvrages hydrauliques transversaux a été totalement reconsidérée. Ce changement est lisible à l'échelle internationale, mais son enregistrement à l'échelle locale en Europe et particulièrement en France suscite de très nombreux débats impliquant l'ensemble des acteurs de la gestion de l'eau (Barraud et Ménanteau, 2009 ; Lejon et al., 2009 ; Germaine et Barraud, 2013). La variabilité des niveaux d'acceptabilité sociale de cette mesure d'ingénierie écologique conduit à s'interroger sur les modèles de gestion des vallées.

$C^{\prime}$ est à partir des rivières de l'ouest de la France ${ }^{2}$ que les enjeux liés à la gestion de cet abondant héritage témoin de l'intensité de l'exploitation de cours d'eau de petite dimension (ordre 2 à 6 selon la classification de Strahler) pour la production d'énergie, la navigation ou encore la mise en valeur agricole des fonds de vallées (Lespez et al., 2005 ; Le Cœur et Gauthier, 2005 ; Barraud, 2007 ; Cador et Lespez, 2012) - sont traités. Rectilignes ou à méandrage libre, ces cours d'eau correspondent à des systèmes fluviaux de faible énergie dont la puissance spécifique est souvent inférieure à $30 \mathrm{~W} / \mathrm{m}^{2}$ (Adam et al., 2007 ; Lespez, 2012). Essentiellement rurales et agricoles, les vallées associées présentent des paysages diversifiés qui rompent la monotonie des plateaux et collines environnants et s'imposent comme des éléments majeurs à l'échelle de l'ouest de la France (Germaine, 2009; Bourget et Le Dû-Blayo, 2010). La gestion de ces vallées se situe à une étape charnière liée à la fin d'un système, celui des moulins et de la rivière aménagée, aujourd'hui révolu mais dont l'influence sur le fonctionnement des hydrosystèmes et la physionomie des paysages reste importante (Lespez et al., 2012). Deux grands modèles de patrimonialisation semblent s'affronter : l'un envisage ces héritages, le moulin à eau et les infrastructures hydrauliques qui lui sont associés (seuils, biefs, vannes) en particulier, comme une ressource à conserver dans le cadre de la valorisation des paysages de vallées, tandis que l'autre les considère comme un obstacle au bon fonctionnement des corridors écologiques. Dans ce modèle, les cours d'eau et zones humides associées sont envisagés comme des infrastructures naturelles linéaires (Mermet, 1995). Apparue dans la sphère institutionnelle de la gestion des milieux (Bernard, 1994), cette notion, reprise par la politique des Trames Vertes et Bleues, a

\footnotetext{
2 Cet article s'appuie sur les réflexions menées dans le cadre de l'ANR JC Reppaval « Représentations des paysages et de la nature dans les petites vallées de l'ouest de la France face aux projets de restauration écologique » (responsable R. Barraud, en collaboration avec M.-A. Germaine, http:/ / reppaval.hypotheses.org/).
}

pour objectif d'élever le statut des zones humides en leur apportant des moyens et une logique de gestion équivalents à ceux mis en œuvre pour d'autres types d'infrastructures d'intérêt général dans la mesure où elles assument des fonctions (climatique, hydrologique, biologique) et rendent des services (ressource en eau, prévention des risques naturels, production de ressources biologiques, valeurs culturelles, touristiques, éducatives, scientifiques et patrimoniales) vitaux pour les sociétés. L'analyse des deux logiques de mise en patrimoine met en exergue les valeurs, mais aussi les états de référence sur lesquels s'appuient ceux qui les promeuvent. Il ne s'agit pas ici de discuter des impacts écologiques des seuils sur les environnements aquatiques, mais de proposer un état des lieux et des pistes de réflexion pour la mise en œuvre de projets de rivières conciliant enjeux écologiques et développement local.

Dans un premier temps, l'inventaire et l'analyse de l'arsenal réglementaire mis en place récemment aux échelles européenne et nationale conduisent à suivre le changement de paradigme de gestion des cours d'eau qui s'opère en France depuis les années 1990. Celui-ci est ensuite mis en regard du modèle techniciste de la rivière aménagée et du modèle écologique émergeant. Leur confrontation permet enfin de proposer de nouvelles perspectives pour la mise en œuvre de projets de rivières partagées.

\section{Gérer et restaurer les rivières : un contexte nouveau}

\section{De l'approche hydraulicienne à la gestion physique}

Le démantèlement des seuils s'inscrit dans la mise en place d'un nouveau paradigme d'aménagement des cours d'eau que souligne l'évolution récente du contexte réglementaire (Encadré). La gestion est dorénavant informée par la science écologique, ce que J.-G. Wasson appelait de ses vœux en 1992. Cette approche scientifique intègre les avancées de l'hydromorphologie et les connaissances sur la chaîne processus/dynamique des habitats/biodiversité/fonctionnalité des milieux qui permettent d'apprécier plus finement les pressions enregistrées par les hydrosystèmes. Cette nouvelle approche est combinée d'une manière finalisée aux principes de l'écologie de la restauration qui reposent sur l'autoréparation des écosystèmes dégradés, voire détruits, pour les reconduire vers leur trajectoire évolutive initiale (Le Floc'h et Aronson, 1995 ; Clewel et Aronson, 2010). Il ne s'agit donc plus seulement d'adopter des pratiques de gestion garantissant le maintien des fonctionnalités des cours d'eau, mais d'intervenir directement sur la structure des milieux fluviaux (morphologie, régime hydrologique, transit sédimentaire) afin de rétablir la 
Encadré. Gestion physique des cours d'eau et restauration de la continuité écologique : rétrospective et contexte actuel

\section{A / Émergence de la question de la continuité écologique}

1851 : Circulaire ministérielle du 23 octobre 1851 à laquelle est annexé un règlement d'eau type à respecter comportant la disposition « passe à poisson » (elle ne sera appliquée que rarement, sur quelques cours d'eau fréquentés par de grands migrateurs tels que le saumon).

1856 : Le barrage/seuil bonifie la rivière : « Il y a entre une rivière dans son état primitif et une rivière pourvue de barrages la même différence qu'entre la nature brute et la nature cultivée, qu'entre un arbre sauvage et un autre chargé de fruits » (Nadault de Buffon).

1865 : Loi du 31 mai 1865 qui introduit la possibilité d'aménager des échelles à poissons sur les barrages des usines hydrauliques à la suite d'une enquête instruite par les conservateurs des Eaux et Forêts (Cazeils, 2003). L'usine du Bazacle sur la Garonne à Toulouse est alors déjà équipée d'une passe à poissons. Une première échelle à poissons avait été mise au point par l'écossais Smith en 1837.

1913 : Aux États-Unis, J. Muir perd la bataille contre la construction du grand barrage de Hetch Hetchy (Yosemite). Cette défaite constituera une pierre angulaire de la structuration du mouvement préservationniste et de la lutte contre les barrages. Cet ouvrage fait actuellement l'objet d'un projet de démantèlement.

\section{B / Les prémices d'une gestion « écologique » fondée sur l'hydromorphologie et l'hydrobiologie}

1980 : Classement « rivières réservées ». Des sections de cours d'eau sont préservées de la construction de nouveaux aménagements hydroélectriques.

1984 : Loi «pêche ». Obligation d'efficacité des dispositifs de franchissement piscicole, obligation d'aménagement d'ouvrages dans les cinq ans qui suivent la publication d'un arrêté « espèces » sur les sections désignées.

Au début des années 1990 : Le démantèlement des seuils et barrages commence à s'imposer comme un outil de restauration écologique aux États-Unis et en Australie en particulier.

1992 : Nouvelle loi sur l'eau. Mise en place du paradigme scientifique de la gestion écologique intégrée (Wasson, 1992).

1995/1996 : Le barrage de Kernansquillec est démantelé dans la vallée du Léguer (Côtes-d'Armor) ; entre 1988 et 2007, cinq ouvrages sont supprimés dans le bassin de la Loire.

2000 : Le rapport de la Commission internationale des grands barrages reconnaît le démantèlement comme une option envisageable.

\section{C / Nouveau droit, nouvelle doctrine de gestion : une mise en œuvre complexe}

2000 : La directive-cadre européenne sur l'eau consacre l'idée de la rivière « infrastructure écologique » fournissant des aménités. L'atteinte du bon état écologique est conditionnée par la restauration des processus hydromorphologiques. En France, Stroffek et Piégay formalisent la notion de " gestion physique ».

2004/2005 : Étude de diagnostic des masses d'eau (DCE). Le compartiment hydromorphologique et l'incidence des seuils deviennent des éléments majeurs de l'expertise institutionnelle (Agence de l'eau, Conseil supérieur de la pêche puis Onema, Fédération de pêche, etc.).

2006 : Loi sur l'eau et les milieux aquatiques (Lema). Elle rénove l'organisation institutionnelle de la gestion de l'eau (création de l'Onema) et se dote d'outils en vue d'atteindre en 2015 l'objectif de «bon état » fixé par la DCE et de reconquérir la qualité écologique des cours d'eau (continuité écologique, obligation d'un débit minimum imposé au droit des ouvrages hydrauliques).

2007 : Le règlement européen « Anguille » 1600 ouvrages (seuils pour la plupart) sont ciblés pour un aménagement prioritaire.

2009: La loi de programmation du Grenelle de l'environnement fixe des objectifs de restauration de la continuité écologique par la mise en place des Trames Vertes et Bleues. L'engagement $114 \mathrm{du}$ Grenelle (restauration des continuités pour les écosystèmes d'eau douce) indique des objectifs précis. Ainsi, ce sont plus de 2000 ouvrages qui devront être « traités » d'ici fin 2015 dont environ 1200 d'ici fin 2012. Le lancement du Plan national de restauration de la continuité écologique s'accompagne de l'annonce symbolique de la suppression des barrages de Vezins et de la Roche-qui-Boit sur la Sélune (Manche). 
Encadré. Suite.

2010 : Circulaire du 25 janvier 2010 qui précise les modalités de mise en œuvre par l'État et ses établissements publics du plan d'action de restauration de la continuité écologique. Attaquée en Conseil d'État par les associations de riverains et de sauvegarde du patrimoine, elle fait l'objet en 2012 d'une mission d'expertise conduite par le Conseil général de l'environnement et du développement durable.

2010/2012 : L'Onema met en ligne un recueil d'expériences françaises de démantèlement et d'aménagement d'ouvrages (seuils) et publie un manuel d'hydromorphologie.

Les éclairages internationaux sont indiqués en grisé.

Document réalisé par R. Barraud et M.-A. Germaine.

dynamique fluviale garante de la diversité biologique (Malavoi et Bravard, 2010). La gestion écologique des cours d'eau est consacrée par le droit à l'échelle européenne (directive-cadre sur l'eau [DCE], 2000) puis nationale (loi sur l'eau et les milieux aquatiques, 2006). Le texte européen est décisif dans la mesure où il soustend des objectifs de reconquête de la qualité de l'eau et impose le respect d'un échéancier précis.

\section{Restauration de la continuité écologique, un outil stratégique pour l'atteinte du bon état des eaux}

La définition et la mise en œuvre des mesures adaptées à ces objectifs de restauration écologique et in fine à la récupération des services écosystémiques assurés par les cours d'eau (Dufour et Piégay, 2009; Amigues et Chevassus-au-Louis, 2011) reposent sur une série de diagnostics prescriptifs. Les référentiels techniques mettent en avant la prépondérance des contraintes hydromorphologiques dans la qualité globale des milieux aquatiques et pointent plus précisément le rôle négatif des seuils (Malavoi et Bravard, 2010 ; Onema, 2010). Des manuels de restauration hydromorphologique des cours d'eau notamment produits par les experts du pôle Onema $^{3} /$ Cemagref ${ }^{4}$ proposent plusieurs solutions techniques pour atténuer ces impacts (Malavoi et Adam, 2007 ; Malavoi et Salgues, 2011), tout en mettant en exergue les plus radicales qui « consiste[nt] à supprimer les seuils (dérasement) ou au moins à en réduire considérablement la hauteur (arasement) » (Adam et al., 2007, p. 38). La restauration de la continuité écologique rétablissant le transport des sédiments et la circulation des poissons s'impose alors comme un outil phare de la reconquête du bon état écologique des rivières. Le détail de l'intégration législative de la nouvelle conception écologique de gestion des cours d'eau et les incidences sur

\footnotetext{
3 Office national de l'eau et des milieux aquatiques.

4 Centre national du machinisme agricole, du génie rural, des eaux et des forêts, devenu l'Institut de recherche en sciences et technologies pour l'environnement et l'agriculture (Irstea).
}

les moyens d'action retenus sont présentés dans l'encadré ci-dessus.

\section{La confrontation de deux modèles de gestion des rivières}

\section{La rivière comme infrastructure naturelle}

La restauration de la rivière en tant qu'infrastructure naturelle, fondée sur la mesure-clé de l'effacement des seuils et barrages, constitue un prolongement de la contestation de l'impact des grands barrages. Depuis le début des années 2000 (CMB, 2000), il s'agit de « libérer les rivières » comme l'illustre le programme Living Rivers du $W_{W W}{ }^{5}$ pour la promotion de la gestion durable des fleuves. L'action est portée par de nombreuses ONG, en Espagne par exemple où le WWF a proposé en 2009 une liste de vingt ouvrages à araser dans une campagne-choc intitulée "Liberando Ríos». En France, des associations internationales (WWF, NASF, ERN) et des associations nationales emblématiques (SOS Loire Vivante, Fondation Nicolas Hulot, FNE) ont milité pour le démantèlement des barrages de Poutès sur la Loire ou de Vezins sur la Sélune.

Dans les faits, c'est aux États-Unis que la politique la plus active d'effacement d'ouvrages est menée (Barraud, 2012). Le mouvement de «dam removal» $\mathrm{s}^{\prime} \mathrm{y}$ est clairement accéléré dans les années 1990 : entre 1912 et 2012 , ce sont près de 1100 ouvrages qui ont été démantelés dont la plupart (800) durant les vingt dernières années selon American Rivers ${ }^{6}$. Les projets les plus emblématiques concernent de grands barrages (Elwah, Washington ou Sandy River, Oregon) mais la plupart des ouvrages arasés sont inférieurs à $10 \mathrm{~m}$ de haut et correspondent à d'anciennes chaussées de moulins. La multiplication des expériences a alimenté de nombreux rapports et une abondante littérature grise qui tentent en particulier de répondre aux difficultés techniques mais

\footnotetext{
5 Fonds mondial pour la nature.

6 http://www.americanrivers.org/initiative/dams/projects/ 2012-dams-removed/
} 
aussi sociales qui sont apparues (American Rivers, 2002 ; David et Baish, 2002 ; Graf, 2003). Ces expériences sont relayées par le réseau International Rivers ou European Rivers Network en Europe (campagne « Dam Decommissioning », co-organisation des campagnes «Elbe Vivante » et « Loire Vivante »). Sur le modèle des grandes associations nord-américaines, SOS Loire Vivante, qui mène depuis 1986 un combat pour la "Loire sauvage » et depuis 1994 pour des "rivières vivantes » partout, ou plus récemment France Nature Environnement (2008) s'engagent dans des campagnes pour le démantèlement des grands comme des petits barrages.

Parallèlement à l'intégration institutionnelle de la stratégie de restauration hydromorphologique des cours d'eau fondée, en particulier, sur l'arasement, l'effacement complet, l'aménagement des ouvrages transversaux ou encore la modification des modes de gestion hydraulique, on assiste à l'émergence d'un mouvement de promotion des rivières "sauvages ». Il est illustré en France par la création du réseau Rivières Sauvages en 2011. Fondé et soutenu par des associations de défense de la nature (WWF, Mountain Wilderness, Conservatoire National Saumon Sauvage, SOS Loire Vivante) et des représentants de la pêche, ce réseau implique également des acteurs institutionnels (Onema, Cemagref). L'association et le fonds associé ont pour ambition de susciter le développement d'une "culture de la nature » inspirée de celle identifiée aux États-Unis, au Québec ou en Irlande afin de protéger efficacement les «dernières rivières naturelles " 7 . Définie comme un " écosystème aquatique au stade climacique» (Rivières sauvages, 2011, p. 22), la « rivière sauvage» se distingue par un label décliné en trois niveaux selon son « degré de naturalité » ou de « sauvagitude », déterminé selon des critères biologiques, hydrauliques, hydro-géomorphologiques et physicochimiques. Ce modèle s'ancre sur l'idée nordaméricaine de Wilderness ou idéologie de la nature vierge (Arnould et Glon, 2006 ; Blandin, 2009). Les images associées privilégient des rivières à méandres, voire en tresses, caractérisées par des écoulements rapides sur fond caillouteux et une succession d'habitats variés insérés dans un environnement verdoyant de prairies humides ou de bois. À moyen terme (dix à quinze ans), la sanctuarisation est clairement reconnue comme un objectif devant concerner près de $10 \%$ du linéaire. Dans l'ouest de la France, cela concerne deux cours d'eau bretons : le Léguer dont les gestionnaires ont demandé le label et l'Ellé identifié comme un site potentiel.

Centré sur l'interprétation écologique, ce modèle considère la vallée comme une infrastructure naturelle à restaurer et appréhende les aménagements hydrauliques systématiquement comme des facteurs de dégradation des milieux, voire des éléments de banalisation du

7 http://rivieres-sauvages.fr/ paysage. Il sous-tend la valorisation d'une esthétique de nature qui puise à la fois dans l'imagerie d'un idéal théorique, un paysage de manuel, mais aussi dans l'imaginaire de la rivière sauvage à forte énergie. Informé par la science, il reste associé à un modèle de représentation de la belle rivière support d'activités et d'une certaine expérience de la nature (Tremblay, 2005). En creux, ce modèle charge de valeurs (esthétique, patrimoniale) négatives le paysage des rivières aménagées (Schnitzler et Génot, 2012 ; Fel, 2009). Les références aux expériences nordaméricaines véhiculent des représentations en décalage avec la réalité écologique, paysagère et culturelle des rivières européennes. Sur le plan opérationnel, l'utilisation de modèles de restauration basés sur les capacités autorégulatrices des systèmes fluviaux à forte énergie pose particulièrement problème à l'échelle de l'ouest de la France (Lespez et al., 2012).

\section{La patrimonialisation de la rivière aménagée}

À l'échelle de l'Europe de l'Ouest, les petites vallées partagent un héritage commun : partout seuils, moulins, biefs, déchargeoirs et autres fossés d'irrigation jalonnent les fonds de vallées. C'est l'ensemble des fonds de vallée de l'ouest de la France qui est aménagé jusqu'au $\mathrm{XIX}^{\mathrm{e}}$ siècle où progrès technique et révolution énergétique mettent fin à cet aménagement (Cador et Lespez, 2012). Cette trame d'ouvrages hydrauliques subsiste à des niveaux de conservation variés, mais la rémanence des formes est assez forte pour peser d'une manière non négligeable sur la gestion actuelle (Barraud, 2007; Lespez, 2012).

Le maintien d'une grande partie de ces aménagements sous-tend l'existence de puissants processus de requalification comme le montrent les analyses diachroniques menées sur les vallées sud-armoricaines et bas-normandes (Barraud, 2008 ; Germaine et al., 2012). La transformation du paysage productif initial en paysage d'agrément a constitué un processus très efficace témoignant de nouveaux modes d'utilisation et d'appropriation de l'espace (résidentielle, récréative, touristique, patrimoniale). Ces requalifications peuvent motiver une restauration du paysage productif du moulin (conservation du plan d'eau et/ou de la chute, remise en état de la roue voire du moulin, etc.). Dans de nombreux cas, la patrimonialisation se traduit par une forte évolution des modes de gestion hydraulique (disparition des roues, abandon des biefs, des vannages, etc.) du fait d'importantes charges d'entretien. La valorisation du patrimoine comme ressource justifie à l'inverse leur maintien (valorisation des seuils par exemple : esthétique de la chute, ambiance sonore). Les héritages requalifiés structurent une rivière-ressource fondée sur l'agrément, la mise en scène de l'histoire locale et la valorisation touristique comme le suggère le cas de la 
Sèvre nantaise (Barraud, 2008). Malgré quelques conversions réussies en écomusée et la création de « routes des moulins », la majorité des héritages correspond cependant à des propriétés privées disséminées dans des fonds de vallées souvent peu accessibles. Le patrimoine est aussi utilisé comme prétexte pour poursuivre des usages qui réclament le maintien des ouvrages ou du moins de la rivière à plein bord et des retenues d'eau. C'est le cas des activités récréatives inscrites tant sur l'eau (canotage, canoë-kayak, pêche, baignade) qu'au bord de l'eau (randonnée, chemin de halage, guinguette). Le propriétaire de moulin est le garant de cette réinvention du paysage productif.

L'essor de la microturbine constitue un autre moteur de la requalification des ouvrages pour près de 2000 microcentrales. La petite hydraulique, qui participe pour $1,5 \%$ à la production électrique nationale, est soutenue par l'État dans le cadre de la promotion des énergies renouvelables. Néanmoins, le renforcement permanent des contraintes techniques (passes à poisson, élévation du débit réservé), donc des investissements, et les difficultés de renouvellement de concessions fragilisent cette activité selon ses promoteurs. L'ambivalence des préconisations du Grenelle a conduit le ministère de l'Écologie à mener une réflexion sur l'optimisation du parc (Convention pour une hydroélectricité durable, 2010) alors que l'Onema est impliqué dans des programmes de recherche couplant évaluation des effets des obstacles sur les migrations piscicoles et mise au point d'équipement moins agressifs sur le plan écologique. La contribution de la petite hydraulique aux énergies vertes et les retombées économiques associées figurent parmi les arguments mis en avant par les défenseurs de la rivière aménagée.

$\mathrm{Au}$ final, de nombreux ouvrages hydrauliques font l'objet d'un usage et/ou d'un fort attachement local. Le modèle défendu par les propriétaires, riverains et collectivités s'ancre sur des valeurs patrimoniales construites à partir des empreintes et de la mémoire des usages du passé. Il s'appuie sur la défense d'une symbolique bâtie à petite échelle mais qui intègre des valeurs régionales et locales. Ce modèle consacre le motif du moulin à eau, avec son seuil (ou bief) et les retenues d'eau relativement profondes et sans courant associées, qui constitue une forme conservatoire du rapport à la rivière. Le lobby de la petite hydroélectricité n'hésite pas à utiliser cette imagerie tandis que les associations de défense du patrimoine diffusent une image idéalisée de la belle et saine rivière à moulins. Celle-ci s'appuie sur le récit d'un âge d'or souvent débarrassé de l'âpreté des rapports sociaux qui caractérisait ces espaces. La référence à l'archive est mobilisée pour revendiquer un paysage en place depuis des temps immémoriaux réglé par le droit et par la science de l'ingénieur et validé par la sédimentation des savoirs vernaculaires. Cependant, ce modèle est aussi mobilisé pour défendre des intérêts particuliers: le maintien sans contrepartie d'un patrimoine strictement privé ou d'usages individuels (irrigation, pêche, agrément). Le discours des défenseurs des moulins se détache de la seule dimension patrimoniale pour promouvoir le paysage d'une rivière à plein bord et de plans d'eaux dont les qualités et usages potentiels sont mis en avant. Ces arguments nouveaux figurent dans les actes des Journées nationales de la FFAM ${ }^{8}$ (2010), significativement intitulées "Pour des rivières vivantes". Dans ce cadre, une allusion à la Convention européenne du paysage (CEP) [Conseil de l'Europe, 2000], mobilisée comme texte de référence engageant la France à préserver partout la qualité des paysages conformément aux aspirations des populations, est intéressante dans la mesure où elle met en avant les divergences entre enjeux écologiques et paysagers comme les déséquilibres entre politiques (DCE, CEP) et échelles associées.

\section{Des injonctions de l'État, relais des campagnes en faveur de l'effacement des barrages ?}

L'interprétation de la DCE, qui fixe des obligations de résultat sans définir les moyens, s'opère de manière distincte dans les pays européens. Ainsi, les opérations de restauration hydromorphologique des masses d'eau menées jusque-là au Royaume-Uni (RRC, 2010 et 2011) par exemple consistent essentiellement à rétablir les espaces de mobilité des cours d'eau. Le rétablissement de la continuité écologique s'opère par des aménagements ou par le démantèlement d'obstacles de petite dimension (RRC, 1999 ; projet Walphy en Belgique). La poursuite à l'échelle européenne de l'inventaire des opérations de restauration des cours d'eau à l'image du recensement mené par la Clearing House of Dam Removal Information aux États-Unis permettrait de mieux appréhender les spécificités locales, mais aussi d'identifier les facteurs de réussite. Le projet Restore (LIFE, European Centre for River Restoration) et l'inventaire des opérations de restauration établi par l'Onema pallient en partie ce manque de suivi observé par B. Morandi et H. Piégay (2011) dans l'ensemble de l'espace français.

En France, le démantèlement des seuils est souvent privilégié dans les discours et dans la littérature grise. À ce parti pris s'ajoute le rôle ambigu de l'expertise institutionnelle qui produit des diagnostics de plus en plus prescriptifs. Ces diagnostics qui visent à identifier les ouvrages à supprimer/modifier en priorité ne sont pas toujours établis en concertation avec les acteurs locaux. À ce titre, la publication de la liste des ouvrages Grenelle par les services de l'État a contribué à mettre sous tension de nombreux syndicats de rivière engagés dans des démarches de diagnostic à l'échelle locale. Les tentatives

Fédération française des amis des moulins. 
d'évaluation collective encouragées à un moment par l'Onema et les agences de l'eau comme celles menées dans les vallées de la Sèvre Nantaise et du Thouet (Barraud et al., 2009) ont, elles aussi, révélé les problèmes de compatibilité entre les approches des experts et les décisions proposées à partir d'une connaissance plus fine du terrain des acteurs et des usages. Par ailleurs, les diagnostics réalisés à l'échelle nationale sont encore incomplets comme l'illustre l'inventaire des obstacles transversaux. La production hydroélectrique, activité de valorisation économique directe des ouvrages, est quasiment la seule à être reconnue : les autres usages (sociaux, récréatifs, patrimoniaux) mal connus et mal évalués sont négligés. De ce fait, seuls $10 \%$ des petits ouvrages sont considérés comme exploités économiquement (Malavoi, 2003) et l'Onema (2010) considère que, parmi les 60000 ouvrages recensés, «plus de la moitié n'ont pas d'usage avéré».

En outre, peu de projets de restauration de cours d'eau menés en France s'appuient sur une participation active du public telle que le prévoit pourtant la DCE (European Union CIS, 2003 ; HarmoniCOP, 2005). Le dispositif de gouvernance paraît à ce jour peu propice au développement de projets de gestion et de mise en valeur coproduits par usagers et experts. Les acteurs locaux se trouvent dans une position paradoxale. Le dispositif de gouvernance paraît à ce jour peu propice au développement de projets de gestion et de mise en valeur coproduits par les usagers et les experts. Les acteurs locaux se trouvent dans une situation paradoxale. En effet, l'État incite les collectivités locales à prendre en charge les compétences de gestion de l'eau en se fédérant (établissements publics territoriaux de bassin). Mais par ailleurs les injonctions nationales en matière d'aménagement des cours d'eau (identification des ouvrages prioritaires, etc.) contraignent de plus en plus les initiatives prises à l'échelle locale. Alors que la question de l'état morphologique des cours d'eau semble peu intégrée par les usagers, comme l'a montré la consultation nationale menée par l'Agence de l'eau en 2008 et 2009, sur le terrain, les gestionnaires doivent assumer, le plus souvent seuls, les incohérences et changements abrupts d'orientation dans la mise en œuvre des politiques publiques de gestion.

\section{Pour de véritables projets de territoire construits autour d'une rivière partagée}

\section{Favoriser la concertation pour le maintien du caractère multifonctionnel des rivières}

Sur le terrain, les gestionnaires sont confrontés à la nécessité d'articuler plusieurs formes de patrimonialisation des vallées (préservation de la nature ou des héritages culturels). Le renouvellement et la recrudescence des conflits sur la scène locale traduisent les difficultés d'arbitrage entre ces modes de mise en patrimoine. La contestation prend des formes diverses (constitution d'associations, affichage, pétitions, articles dans la presse locale, manifestations). À travers celle-ci, il s'agit parfois de défendre un patrimoine historique, architectural ou industriel, comme cela a pu être le cas en 1996 lors de l'effacement du barrage de Kernansquillec (Côtes-d'Armor) où les habitants ont exprimé un attachement fort au barrage et surtout à la papeterie Vallée, véritable symbole de la mémoire locale (MEDD, 2002). Toutefois, la contestation locale exprime aussi la défense plus générale d'un cadre de vie et d'aménités associées. C'est ce qu'expriment les pétitions et protestations des riverains de la Maine et de la Sèvre au cours des expérimentations d'ouverture des vannes des barrages menées par le syndicat Sevraval durant l'été 2011, mais également les associations créées dans la vallée du Don en Loire-Atlantique ("Touche pas à ma rivière »), dans les vallées de la Jouanne de l'Oudon, etc. Plus que les ouvrages, c'est le paysage associé composé d'une rivière à plein bord, c'est-à-dire d'un cours d'eau remplissant totalement son chenal jusqu'au sommet des berges, et de plans d'eau que les habitants souhaitent conserver. Les arguments utilisés par l'association de propriétaires en opposition à tout abaissement de niveau du Thouet et médiatisés dans la presse quotidienne régionale révèlent la prégnance des usages privatifs (irrigation, populiculture, arrosage des jardins, réserve incendie), mais elle confirme surtout que la beauté des plans d'eau demeure le premier argument invoqué pour plaider leur maintien. Les conflits observés tendent à illustrer les divergences entre deux principales logiques de conservation, celle du paysage et des aménités d'un côté, celle de l'environnement et de la biodiversité de l'autre (Germaine et Barraud, 2013).

Le jeu des acteurs mobilisés pour lutter contre ou pour des opérations de restauration est toutefois plus complexe, ces projets divisant au sein même de groupes apparemment homogènes. Les défenseurs de la nature sont partagés entre protection de la biodiversité et valorisation des énergies renouvelables. En Normandie, le maintien de microcentrales pourtant encore en activité est rendu difficile par l'évolution du contexte juridique : l'autorisation délivrée par l'État n'a pas été renouvelée pour l'une d'entre elles et quatre autres arrivent à échéance. Ces ouvrages situés sur la Vire et la Sienne sont sur des axes migrateurs d'intérêt majeur du SDAGE $^{9}$ Seine-Normandie et inscrits au Plan de gestion anguille depuis 2007. Les projets d'effacement de seuils divisent également les pêcheurs. Si la position nationale, représentée par la Fédération nationale, est favorable à la restauration de la continuité écologique, des clivages

\footnotetext{
Schéma directeur d'aménagement et de gestion des eaux.
} 
apparaissent à l'échelon local. Les pêcheurs sportifs, pêcheurs à la mouche fréquentant les sections d'eaux vives, sont généralement favorables au démantèlement des seuils qui permettrait la remontée des poissons migrateurs vers les frayères. À l'inverse, les pêcheurs de poissons blancs, habitués des eaux lentes caractéristiques des plans d'eau situés en amont des barrages, s'y opposent. Ces oppositions sont renforcées par des différences de profils socioéconomiques. Requérant beaucoup de temps de loisir, de technicité et un équipement coûteux, la pêche à la mouche est considérée comme un « loisir haut de gamme [...] assurant une différenciation sociale marquée » (Bonnieux et Vermersch, 1993), le plus souvent pratiqué par des pêcheurs venus d'ailleurs (Îlede-France et Belgique dans le nord-ouest de la France). À l'inverse, la pêche de détente constitue une activité populaire exercée à proximité du lieu de résidence dans la plupart des cas. Cette différence exacerbe le sentiment de dépossession de la rivière ressenti par les pêcheurs locaux face aux projets de restauration. C'est ce qu'ont exprimé les pêcheurs de la vallée de la Touques en organisant des manifestations dénonçant des projets de restauration pour une pêche réservée aux riches "horsains " ${ }^{10}$ et une privatisation de la rivière (Germaine, 2012). La position du monde de la pêche est d'autant plus complexe que son message est parfois brouillé entre des équipes d'ingénieurs suivant la ligne de l'Onema et des présidents de fédération parfois totalement contre la suppression d'ouvrages.

Ce constat renvoie plus largement à l'inadéquation entre le caractère multifonctionnel des rivières et les modes de gestion univoques promus par une partie des acteurs de l'eau en faisant des fonds de vallées des sanctuaires. Il faut ici insister sur l'hétérogénéité des conceptions d'une bonne gestion des rivières sur lesquelles se fondent les partisans de cours d'eau plus naturels. Les associations de protection de la nature s'appuient sur la seule dimension éthique pour justifier des actions de réparation et de conservation de cours d'eau (ou sections) très peu artificialisés. D'inspiration préservationniste, cette position, défendue par les militants mais aussi partagée par une partie des gestionnaires, met en avant la valeur intrinsèque des milieux non dégradés / modifiés par l'homme. La position institutionnelle appréhende le fond de vallée (le plus souvent le lit majeur) comme une infrastructure naturelle tributaire du fonctionnement d'un bassin versant. Les services de l'État, notamment les agences de l'eau, légitiment leurs programmes d'action (restauration hydromorphologique) en faveur de systèmes "plus naturels » par le fait que ces derniers conditionnent l'atteinte du bon état écologique des masses d'eau (DCE) et garantissent la pérennité des bénéfices rendus aux humains : services

\footnotetext{
10 En normand, désigne un étranger.
}

d'approvisionnement, services de régulation, services culturels, services de soutien (MEA, 2005). Ce sont donc en réalité trois visions de la bonne/belle rivière qui s'affrontent dans le débat sur leur devenir.

Face à la remise en cause d'un certain nombre d'activités, des arbitrages doivent s'opérer. La notion de services écologiques est de plus en plus convoquée et souvent couplée à une évaluation financière pour aider à la prise de décision. Or, la concertation reste globalement absente ou bien située très à l'aval des procédures de décision et les aménités environnementales (bienfaits non matériels que procurent les écosystèmes : bénéfices récréatifs, culturels, esthétiques, pédagogiques, etc.) demeurent peu prises en compte, jamais évaluées financièrement et rarement véritablement mises en débat. L'identification de l'ensemble des services environnementaux (services écologiques et aménités) effectivement rendus par les infrastructures naturelles dans un contexte territorial donné et selon différents scénarios d'aménagement constitue un enjeu scientifique majeur dans lequel les sciences humaines et sociales doivent $\mathrm{s}^{\prime}$ investir. Les agences de l'eau ont pris conscience du poids de l'enjeu de concertation. L'agence LoireBretagne y a consacré un guide méthodologique. Il s'agit en réalité plus d'un outil pour mettre en œuvre des démarches de communication visant à « obtenir l'adhésion des riverains aux projets de restauration des cours d'eau » (agence de l'eau Loire-Bretagne, 2011). Il est d'ailleurs assez significatif que cette phase ne soit envisagée qu'une fois les orientations fixées: «Tant que le projet n'est pas bien défini et clairement porté politiquement, mieux vaut s'abstenir d'organiser une réunion publique » (ibid., p. 30). Les orientations de gestion relèvent donc avant tout de rapports de force. Elles témoignent de la difficile conciliation entre des préoccupations globales, envisagées à un niveau planétaire justifiant des décisions «d'en haut » (Larrère et Larrère, 1997) et des attentes locales ancrées dans la réalité socioéconomique des territoires. Alors que les textes récents, la convention d'Aarhus, la CEP ou même la DCE, rendent obligatoire la prise en compte de la demande sociale, ce constat pose la question de la place de l'expert et du citoyen dans le processus décisionnel et milite pour une analyse approfondie des dispositifs de gouvernance. Dans ce sens, l'expérience britannique est intéressante dans la mesure où le recours à la concertation y est beaucoup plus fréquent (Environment Agency, 2004 ; Halcrow et Geodata Institute, 2009).

\section{Tenir compte des aspects socioéconomiques dans les diagnostics pour des rivières partagées et accessibles}

En outre, bien que ces opérations soient dictées par des problématiques environnementales, elles ont des incidences socioéconomiques et modifient les pratiques 
spatiales des usagers. Or, ces effets restent peu traités dans les expertises commandées par les services de l'État. Le recueil d'expérience sur la restauration hydromorphologique (Onema, 2010) montre clairement à travers 60 exemples d'opérations menées ces vingt dernières années que les volets social et économique sont délaissés (Morandi et Piégay, 2011). La réussite des projets dépend de la capacité des collectivités à trouver un groupe d'acteurs animés d'une véritable volonté de valorisation et de développement pour la vallée et capables de s'affirmer pour établir un rapport de force différent dans les orientations de gestion. Cela a pu être le cas, par exemple, lors de l'effacement du plan d'eau du Coupeau sur le Vicoin en périphérie de Laval où les enjeux récréatifs ont été relayés par les élus (Onema, 2010). Parce qu'elle a évité ces écueils, l'expérience menée à Kernansquillec fait figure d'exemple (Derville et al., 2001 ; MEDD, 2002) : la participation effective des habitants tout au long du processus et l'introduction d'objectifs socioéconomiques ont permis de mettre en œuvre un projet alternatif au barrage emportant l'adhésion de la population. De même, une analyse exploratoire des projets de démantèlement ayant suscité une adhésion des riverains et usagers révèle le manque de prise en compte de la diversité des sites hydrauliques considérés et des contextes géographiques/paysagers dans lesquels ils s'inscrivent. Dans la vallée de la Moine, un projet de suppression de six ouvrages localisés pour la plupart dans la traversée urbaine de Cholet fait partie de ces projets fondés sur un haut niveau de concertation qui sont conduits avec un soutien local solide ${ }^{11}$. Ailleurs en Europe, comme au Royaume-Uni, l'attention portée à la multifonctionnalité des cours d'eau conduit à des projets intégrés mêlant restauration écologique et valorisation : le London Rivers Plans (RRC, 2009) sur le bassin de la Tamise est ainsi présenté comme « un outil pour restaurer les rivières pour la population et la nature ».

Enfin, l'accessibilité au cours d'eau demeure négligée malgré les recommandations de la DCE. Si l'eau est considérée comme un patrimoine collectif, la rivière constitue souvent une enclave inaccessible malgré un entretien financé par des fonds publics (Germaine, 2012). Contrairement aux projets menés outre-Manche, où une tradition à la valorisation des cheminements au bord de l'eau et une organisation différente de la protection de la nature semblent avoir permis d'associer restauration écologique et valorisation récréative, les projets menés sur les cours d'eau français ne bénéficient qu'à certaines catégories d'acteurs (pêcheurs à la mouche, défenseurs de l'environnement, propriétaires riverains). Or, la redécouverte des fonctionnalités écologiques a entraîné un nouvel intérêt pour les vallées. En redonnant accès

\footnotetext{
${ }^{11}$ http:/ / www.sevre-nantaise.com/actions/dossier-effacement-de-6-ouvrages-hydrauliques-sur-la-moine-a-cholet
}

visuellement à des rivières jusqu'alors pour beaucoup d'entre elles cachées car abandonnées, les opérations d'entretien ont suscité une réappropriation de ces paysages et éveillé de nouvelles attentes comme cela a été démontré pour la Touques par exemple (Germaine, 2012). Ces transformations paysagères constituent par ailleurs des externalités positives plus faciles à apprécier que l'amélioration de la qualité des milieux aquatiques : elles peuvent dans ce sens participer d'une politique de sensibilisation à l'environnement. Une réflexion sur le partage de la rivière s'impose tout spécialement sur les cours d'eau à poissons migrateurs sur lesquels de fortes pressions s'exercent (pêche privative, morcellement des parcours) ainsi que sur les tronçons soumis à d'importants processus de résidentialisation. L'application des principes du développement durable incite alors à redéfinir le concept de « rivières vivantes » mobilisé par tous mais selon des définitions variables (vie biologique pour les uns, pluralité de services rendus pour les autres, activités socioéconomiques et cadre de vie pour les derniers). Si le caractère multifonctionnel de celles-ci ne peut être conservé partout, il semble important de ne privilégier a priori aucun usage.

\section{Un élargissement d'échelle nécessaire pour passer de l'enjeu écologique à de véritables projets de territoire}

Bien que pertinente dans une large mesure, l'approche dominante actuelle est problématique dans la mesure où elle relègue d'autres modes d'interprétation de l'objet vallée qui, à l'échelle locale, constitue un espace approprié, vécu, rêvé. Ces vallées sont encore fortement structurées par des lieux ancrés dans l'imaginaire et les pratiques collectives. Marquées par des siècles d'activité industrielle, elles sont devenues des lieux d'agrément où s'articulent des logiques d'appropriation de l'espace variées, parfois concurrentes (Pustlenik, 2008). Dans l'ouest de la France, les vallées intérieures avec des sites escarpés telles la Suisse normande ou les Alpes mancelles constituent tout spécialement des lieux privilégiés du dépaysement, et sont aussi des espaces ressource comme cadre de vie et pour le tourisme. Animées, selon une intensité variable, par un tissu complexe d'usages non productifs, les vallées sont sans conteste des infrastructures naturelles mais pas seulement. L'analyse économique classique rend compte d'un espace productif en crise, sur le déclin. Le diagnostic doit intégrer des usages qui ne génèrent pas de valorisation économique directe afin de mieux rendre compte de la réalité de ces espaces. Cela implique un changement d'échelle. Une approche globale replaçant la rivière dans son contexte géographique doit permettre de dépasser les approches sectorielles. Des outils de diagnostic technique pour l'analyse hydroécologique des cours d'eau ont été développés, en particulier par l'Onema (Malavoi 
et Bravard, 2010) et des travaux ont été menés sur la perception des paysages aquatiques (Cottet, 2010), mais il n'existe en revanche pas de méthodes permettant de saisir les vallées dans leurs dimensions sociospatiales (représentations, logiques d'appropriation, rapports de force) et paysagères (aménités). En outre, le paysage, s'il permet une approche plus large que les diagnostics écologiques, est souvent réduit à sa composante esthétique. L'expertise paysagiste est très rarement mobilisée alors qu'elle s'est imposée dans la reconquête des fronts d'eau urbains des grandes agglomérations (Romain, 2010) et qu'elle pourrait jouer un rôle décisif dans la recréation d'espaces publics de bords d'eau dans les traversées de petites et moyennes villes.

\section{Conclusion}

Ainsi, ce n'est probablement pas un modèle de gestion unique qui remplacera le modèle de la rivière aménagée. Si les enjeux environnementaux qui commandent la multiplication des projets de restauration écologique des cours d'eau sont aujourd'hui reconnus et les préconisations qui en découlent acceptées par les collectivités, le modèle des "rivières sauvages " ne peut s'appliquer à l'ensemble du réseau hydrographique. La construction de rivières partagées nécessite d'élargir les diagnostics, à l'échelle de la vallée et vers les volets sociaux et économiques, pour une conciliation entre enjeux écologiques et attentes locales et une meilleure cohabitation des usages. Dans ce cadre, la poursuite des travaux entamés sur les vallées de l'ouest de la France doit permettre de proposer des diagnostics complémentaires aux approches environnementales et de réintégrer les dimensions sociale et temporelle dans les projets de gestion. Les premiers résultats obtenus incitent à placer la question de la multifonctionnalité au cœur de ces derniers et témoignent de la nécessité de travailler en partenariat avec les structures opérationnelles directement concernées par ces enjeux. C'est donc une réflexion approfondie sur la mise en place de politiques de concertation dans le domaine de la gestion des cours d'eau et la création de nouvelles structures d'arbitrage qui est proposée pour développer des démarches innovantes de concertation et de participation afin d'aller vers des rivières partagées. Il s'agit donc de ne plus considérer le fond de vallée ou la vallée comme une infrastructure naturelle, mais bien de les envisager comme des objets géographiques humanisés, construits, représentés et habités. Ce changement d'approche est surtout nécessaire pour passer de la question de la continuité écologique à l'élaboration de véritables projets de territoire qui prennent en compte les problématiques environnementales. Il s'agit de dépasser la restauration écologique pour l'accompagner d'une véritable réflexion sur un projet de rivière/vallée susceptible de participer au développement durable des territoires.

\section{Références}

Adam, P., Debiais, N., Malavoi, J.-R., 2007. Manuel de restauration hydromorphologique des cours d'eau, Direction de l'eau, des milieux aquatiques et de l'agriculture (Demaa), Service eaux de surface, Nanterre, Agence de l'eau SeineNormandie (http://www.eau-seine-normandie.fr/filead$\mathrm{min} /$ mediatheque/Collectivite/HYDROMORPHO/ 01Manuel_restauration.pdf).

Agence de l'eau Loire-Bretagne, 2011. Guide méthodologique. Restauration des cours d'eau: communiquer pour se concerter, (http://www.eau-loire-bretagne.fr/espace_documentaire/ documents_en_ligne/guides_milieux_aquatiques/Guide_ restauration-CE.pdf).

American Rivers, 2002. The Ecology of Dam Removal: A Summary of Benefits and Impacts, Washington (http://www.michigandnr.com/PUBLICATIONS/PDFS/fishing/dams/ecologyofDamRemoval.pdf).

Amigues, J.-P., Chevassus-au-Louis, B., 2011. Évaluer les services écologiques des milieux aquatiques: enjeux scientifiques, politiques et opérationnels, Coll. Comprendre pour agir, Onema (http:/ / www.onema.fr/IMG/pdf/servicesecologiques_ BD.pdf).

Arnould, P., Glon, É., 2006. Wilderness, usages et perceptions de la nature en Amérique du Nord, Annales de Géographie, $649,227-238$.

Barraud, R., 2007. Vers un tiers-paysage? Géographie paysagère des fonds de vallées sud-armoricaines : héritage, évolution, adaptation. Thèse de doctorat de Géographie, Université de Nantes.

Barraud, R., 2008. Approche géographique de l'intentionnalité paysagère dans les vallées de l'ouest de la France (de la fin du XVIII ${ }^{\mathrm{e}}$ siècle à aujourd'hui), Projets de Paysage, 1 (http:// www.projetsdepaysage.fr/fr/approche_geographique_de_ 1_intentionnalite_paysagere_dans_les_vallees_de_1_ouest_ de_la_france).

Barraud, R., 2012. Rivières du futur, wild rivers ?, VertigO, Hors-série 10 (http:/ / vertigo.revues.org/11411).

Barraud, R., Charrier, A., Constantin, O., Charruaud, G., 2009. Restauration écologique et paysagère des rivières à seuils : retour d'expériences (bassin de la Sèvre nantaise et du Thouet, ouest de la France), Ingénieries EAT, $\mathrm{N}^{\mathrm{O}}$ spécial Écologie de la restauration, 17-30.

Barraud, R., Ménanteau, L., 2009. Émergence du paradigme de la gestion physique des cours d'eau et héritages paysagers : exemple des rivières « à moulins » de l'ouest de la France, Revue du Nord Archéologie, $\mathrm{N}^{\mathrm{o}}$ spécial Lit mineur, lit majeur, lit voyageur, 189-203.

Bernard, P., 1994. Rapport d'évaluation sur les politiques publiques en matière de zones humides. Comité interministériel des politiques publiques, Premier Ministre, Commissariat au Plan, La Documentation française, Paris.

Blandin, P., 2009. De la protection de la nature au pilotage de la biodiversité, Versailles, Éditions Quae.

Bonnieux, F., Vermersch, D., 1993. Bénéfices et coûts de la protection de l'eau : application de l'approche contingente 
à la pêche sportive, Revue Économique Politique, 103, 131151.

Bourget, E., Le Dû-Blayo, L., 2010. Cartographie des paysages : apport à l'analyse des trames vertes et bleues : l'exemple du Pays de Saint-Brieuc, Projets de Paysage, 4 (http:/ / www. projetsdepaysage.fr/fr/cartographie_des_paysages_ apport_a_1_analyse_des_trames_vertes_et_bleues).

Cador, J.-M., Lespez, L., 2012. Les cours d'eau bas-normands aujourd'hui : entre hydrosystèmes et systèmes hydrauliques, in Lespez, L. (Ed.), Paysages et gestion de l'eau : sept millénaires d'histoire des vallées en Normandie, Bibliothèque du Pôle rural 3, Presses universitaires de Caen, MRSH Caen, 235-272.

Clewel, A.-F., Aronson, J., 2010. La Restauration écologique, Arles, Actes Sud.

CMB, 2000. Barrages et développement: un nouveau cadre pour la prise de décisions. Rapport de la Commission mondiale des barrages (http:/ / www.unep.org/dams/wcd/report/WCD $\% 20$ report_Barrages $\% 20$ et $\% 20 \mathrm{D} \% \mathrm{C} 3 \%$ A9veloppement_ pr\%C3\%A9face.pdf).

Cottet-Tronchère, M., 2010. La perception des bras morts fluviaux : le paysage, un médiateur pour l'action dans le cadre de l'ingénierie de la restauration. Approche conceptuelle et méthodologique appliquée aux cas de l'Ain et du Rhône. Thèse de doctorat de Géographie, Université Lyon 3 (http:/ / halshs. archives-ouvertes.fr/docs/00/55/51/46/PDF/these.pdf).

David, S.D., Baish, S. (Eds), 2002. Dam Removal: Science and Decision Making, Washington, The H. John Heinz III Center for Science, Economics and the Environment.

Derville, I., Bonenfant, M., Royet, P., Lepetit, D., Jigorel, A., 2001. Retour d'expériences du démantèlement du barrage de Kernansquillec, Ingénieries EAT, 25, 13-27.

Dufour, S., Piégay, H., 2009. From the myth of a lost paradise to targeted river restoration: forget natural references and focus on human benefits, River Research and Applications, 25, 5, 568-581.

Environment Agency, 2004. Ribble Pilot River Basin: Public Participation and River Basin Planning - Early Experiences, South Preston, UK.

European Union, Common Implementation Strategy for the Water Framework Directive, 2003. Public participation in relation to the water framework directive, Guidance document $\mathrm{n}^{\mathrm{o}} 8$ (http://www.waterframeworkdirective.wdd.moa. gov.cy/docs/GuidanceDocuments/Guidancedoc8Publicparticipation.pdf).

Fel, L., 2009. L'Esthétique verte: de la représentation à la présentation de la nature, Seyssel, France, Éditions Champ Vallon.

FFAM, 2010. Pour des rivières vivantes, Journées nationales de la FFAM, 25-26 novembre, Cholet, France.

France Nature Environnement, 2008. La morphologie des cours d'eau, Consultation du public 2008 sur l'eau, Dossier thématique, 2 .

Génot, J.-C., 2003. Quelle éthique pour la nature?, Aix-en-Provence, Édisud.

Germaine, M.-A., 2009. De la caractérisation à la gestion des paysages ordinaires des vallées du nord-ouest de la France: représentations, enjeux d'environnement et politiques publiques en Basse-Normandie. Thèse de doctorat de Géographie, Université de Caen Basse-Normandie.

Germaine, M.-A., 2012. Dépasser l'enjeu piscicole, vers la définition d'une gestion concertée du cours d'eau et de ses berges: l'exemple de la vallée de la Touques (Calvados), Géocarrefour, 86, 3/4, 161-175.

Germaine, M.-A., Lespez, L., Cador, J.-M., 2012. Le poids des héritages dans la gestion durable des paysages des basses vallées côtières de l'ouest de la France, in Lespez, L. (dir.), Paysages et gestion de l'eau: sept millénaires d'histoire des vallées en Normandie, Bibliothèque du Pôle rural 3, Presses universitaires de Caen, MRSH Caen, 273-302.

Germaine, M.-A., Barraud, R., 2013. Restauration écologique et processus de patrimonialisation des rivières dans l'ouest de la France, VertigO, Hors-série 16 (http:/ / vertigo.revues. org/13583).

Graf, W. L. (Ed.), 2003. Dam removal research: Status and prospects, Proceedings of The Heinz Center's Dam Removal Research Workshop, 23-24 October 2002, Washington, The H. John Heinz III Center for Science, Economics and the Environment.

Halcrow, Geodata Institute, 2009. Strategic Framework for the Restoration of the River Avon System. Final Consultation Report (http://www.environment-agency.gov.uk/static/ documents/Leisure/Non_Technical_Summary_Final_ Report.pdf).

HarmoniCOP, 2005. Learning together to manage together, improving participation in water management, Handbook. University of Osnabrück, Institute of Environmental Systems Research, Osnabrueck, Germany.

Larrère, C., Larrère, R., 1997. Du bon usage de la nature : pour une philosophie de l'environnement, Paris, Aubier.

Le Cœur, C., Gautier, E., 2005. « Les enjeux des friches hydrauliques : entretien, ré-affectation, dés-aménagements. Présentation d'un programme de recherche ", in Serna, V., Gallicé, A. (Eds), La rivière aménagée : entre héritages et modernité, Aestuaria, 7, 495-508.

Le Floc'h, E., Aronson, J., 1995. Écologie de la restauration : définition de quelques concepts de base, Natures Sciences Sociétés, Hors-série, 29-35.

Lejon, A. G. C., Malm Renöfält, B., Nilsson, C., 2009. Conflicts associated with dam removal in Sweden, Ecology and Society, 14, 2, 4.

Lespez, L. (Ed.), 2012. Paysages et gestion, de l'eau : sept millénaires d'histoire des vallées en Normandie, Bibliothèque du Pôle rural 3, Presses universitaires de Caen, MRSH Caen.

Lespez, L., Garnier, E., Cador, J.-M., Rocard, D., 2005. « Les aménagements hydrauliques et la dynamique des paysages des petits cours d'eau depuis le XVIII ${ }^{\mathrm{e}}$ dans le nordouest de la France: l'exemple du bassin versant de la Seulles (Calvados) ", in Serna, V., Gallicé, A. (Eds), La rivière aménagée : entre héritages et modernité, Aestuaria, 7, 125-145.

Lespez, L., Viel, V., Cador, J.-M., Germaine, M.-A., GermainVallée, C., Rollet, A.-J., Delahaye, D., 2012. Environmental dynamics of small rivers in Normandy (western France) since the Neolithic era: What lessons for today in the context of the European Water Framework Directive?, in ArnaudFassetta, G., Reynard, E. (Eds), Continental Hydrosystems and 
Territory and the Different Laws and Practices of Water Management in Europe, Munich, Pfeil.

Malavoi, J.-R., 2003. Stratégie d'intervention de l'agence de l'eau sur les seuils en rivière. Rapport d'étude, AREA-Eau Environnement, AELB.

Malavoi, J.-R., Adam, P., 2007. La restauration morphologique des cours d'eau : concepts et principes de mise en œuvre, Ingénieries EAT, 50, 49-61.

Malavoi, J.-R., Bravard, J.-P., 2010. Eléments d'hydromorphologie fluviale, Coll. Comprendre pour agir, Onema (http:// www.onema.fr/hydromorphologie-fluviale).

Malavoi, J.-R., Salgues, D., 2011. Arasement et dérasement de seuils : aide à la définition de Cahier des Charges pour les études de faisabilité. Compartiments hydromorphologie et hydroécologie. Rapport Onema-Cemagref (http://www.onema.fr/ IMG/pdf/2011_009.pdf).

MEDD, 2002. La renaissance d'une vallée: démantèlement du barrage de Kernansquillec et réhabilitation du site. Ministère de l'Écologie et du Développement durable, Paris.

Mermet, L., 1995. Les infrastructures naturelles : statut, principe, concept, ou slogan ?, Zones Humides Infos, 7, 7-9.

Millenium Ecosystem Assesment (MEA), 2005. Ecosystem Wealth and Human Well Being: Synthesis, Island Press, Washington.

Morandi, B., Piégay, H., 2011. Les restaurations de rivière sur Internet : premier bilan, Natures Sciences Sociétés, 19, 3, 224235.

Onema, 2010. La restauration des cours d'eau : recueil d'expériences sur l'hydromorphologie (http://www.onema.fr/Hydromorphologie,510).
Pustelnik, G., 2008. Les établissements publics territoriaux de bassin, pivots de l'action territoriale pour une gestion équilibrée de l'eau et des milieux aquatiques à l'échelle des bassins versants, Revue des Sciences de l'Eau, 21, 2, 199-218.

River Restoration Centre, 1999. The Manual of River Restoration Technique, Cranfield, UK.

River Restoration Centre, 2009. Proceedings of the $10^{\text {th }}$ Annual RRC Network Conference, 1-2 April, University of Nottingham, UK.

River Restoration Centre, 2011. Building with nature: Managing rivers at the local and catchment scale, $12^{\text {th }}$ Annual Network Conference, 14 April, (http://www.therrc.co.uk/rrc_ conferences.php).

Rivières sauvages, 2011. Le réseau des rivières sauvages : étude de préfiguration (http://www.mountainwilderness.fr/IMG/ pdf/rapport_riviere_sauvage_2010.pdf).

Romain, F., 2010. La construction contemporaine des paysages fluviaux urbains : le cas de deux villes méditerranéennes : Perpignan et Montpellier. Thèse de doctorat en Sciences et Architecture du paysage, AgroParisTech/ENSP, Versailles.

Schnitzler, A., Génot, J.-C., 2012. La France des friches de la ruralité à la féralité, Versailles, Éditions Quae.

Tremblay, F., 2005. Du « pas dans ma cour » à l'écocitoyenneté : opposition aux centrales hydroélectriques privées, Nouvelles Pratiques Sociales, 18, 1, 26-38.

Wasson, J.G., 1992. Les orientations fondamentales par bassin: propositions pour une gestion intégrée des écosystèmes d'eau courante, Cemagref, Division BEA, LHQ, Lyon.

WWF Espagne, 2009. Liberando Ríos : propuesta de WWF para el desmantelamiento de presas en Espana, Madrid (http:// assets.wwf.es/downloads/presas_informe_completo.pdf).

Reçu le 14 juin 2011. Accepté le 7 novembre 2012. 